Voix et Images

\title{
L'édition critique de Maria Chapdelaine par Ghislaine Legendre
}

\section{Guy Laflèche}

Volume 8, numéro 1, automne 1982

Madeleine Gagnon

URI : https://id.erudit.org/iderudit/200366ar

DOI : https://doi.org/10.7202/200366ar

Aller au sommaire du numéro

Éditeur(s)

Les Presses de l'Université du Québec

ISSN

0318-9201 (imprimé)

1705-933X (numérique)

Découvrir la revue

Citer cet article

Laflèche, G. (1982). L'édition critique de Maria Chapdelaine par Ghislaine

Legendre. Voix et Images, 8(1), 61-82. https://doi.org/10.7202/200366ar d'utilisation que vous pouvez consulter en ligne.

https://apropos.erudit.org/fr/usagers/politique-dutilisation/ 


\title{
L'édition critique de Maria Chapdelaine par Ghislaine Legendre
}

\author{
par Guy Laflèche, Université de Montréal
}

\section{1 - UN ROMAN POUR ADOLESCENTS}

Tout le monde (le monde occidental en tout cas) connaît au moins de nom Maria Chapdelaine, le "récit du Canada français" du romancier français Louis Hémon, mort «en pleine tempête de neige " (le 8 juilletl) à Chapleau, en Ontario, après un séjour d'un peu moins de deux ans au Québec loctobre 1911 juillet 1913). Ėcrit pour les Français, Maria Chapdelaine est un extraordinaire et machiavélique roman pour adolescents. La droite française nous en a fait un «chef-d'ceuvre sur le Canada», selon le titre de l'article de Léon Daudet paru dans l'Action française de Maurras le 8 juin 1921. Henri Massis n'aura pas de peine à nous en convaincre en présentant le roman dans la Croix, le 11 août 1921, sous le titre «Maria Chapdelaine, un chef-d'œuvre catholique». Nos intellectuels et Damase Potvin trouveront l'idée excellente, de sorte que petit à petit le chef-d'œuvre catholique sur le Canada est devenu un chefd'œuvre de littérature québécoise. Cela n'a pas été trop difficile, car tout le monde sait que la littérature québécoise ne comprend que des chefs-d'œuvre. Pas des romans de collégiens, du Chercheur de trésors jusqu'à la Famille Plouffe, pas des romans complètement niaiseux comme la Terre paternelle et Un homme et son péché, ni même d'excellents romans populaires comme Bonheur d'occasion. Non, la littérature québécoise n'est faite que d'immortels chefs-d'œuvre. Maria Chapdelaine aura donc été le chef-d'œuvre de noš chefs-d'œuvre. Notre chef-d'œuvre de portée internationale, dont il suffit de dire aujourd'hui que "l'époque de Maria Chapdelaine est bien entendu révolue». Exactement comme si l'on s'amusait à présenter la France ou sa littérature en parlant de l'époque bien entendue révolue d'À la recherche du temps perdu de Proust. Tout cela constitue le Mrthe de Maria Chapdelaine étudié par Nicole Deschamps, Raymonde Héroux et Normand Villeneuve dans le livre paru sous ce titre aux Presses de l'Université de Montréal en 1980. Jean Royer en a fait le compte rendu dans Le Devoir du 18 octobre 1980 (pp. 21 et 40 ). Ce mythe est bien entendu en rapport dialectique avec le succès de librairie des éditions Bernard Grasset qui en ont fait un best-seller: Gabriel Boillat a présenté une saisissante chronologie de ce phénomène dans la Revue d'Histoire littéraire de France en mars-avril 1974 (pp. 223-253). 


\section{Un roman pour adolescents}

Ce n'est pas du tout par esprit polémique ou pour faire scandale que je dis qu'il s'agit d'un «extraordinaire et machiavélique roman pour adolescents". II y a de très beaux romans pour adolescents et Maria Chapdelaine en est un particulièrement bien réussi. A cause de sa langue, de son style et de son rythme, ce roman est immédiatement accessible à de très jeunes lecteurs (un peu comme l'lliade d'Homère qu'on ne pense pas souvent à leur offrir). A cause aussi de son contenu: I'histoire qu'il raconte, sa valeur poétique, narrative et philosophique. Maria Chapdelaine pose une des premières problématiques de l'âge adulte, celle qui surgit radicalement au moment de l'adolescence et qu'on met ensuite toute sa vie à résoudre. En effet, à travers une histoire pittoresque et apparemment toute régionale, c'est une question universelle et existentielle qui est posée: l'équilibre du principe du plaisir et du principe de la réalité. Nous rêvons tous comme Maria Chapdelaine d'épouser des princes et des princesses et nous épousons tous des hommes et des femmes ordinaires. Il y aura toujours une différence malheureuse et irrémédiable entre rêver d'aimer et aimer, entre rêver et vivre. Maria Chapdelaine rêve d'aimer François Paradis qui représente la magie de la forêt, elle est tentée aussi par Ephrem Surprenant, l'Américain symbolisant toutes les vies qui sont ailleurs, mais elle finira par dire à son voisin, Eutrope Gagnon, qu'elle l'épousera «le printemps d'après ce printemps-ci, quand les hommes reviendront du bois pour les semailles". Cette histoire simple, trop simple, qui se déroule en une quinzaine de tableaux sur une année de la vie paysanne, n'est pourtant pas simpliste: d'abord parce qu'il s'agit de l'histoire de tous les amoureux et de tous les couples du monde et ensuite parce qu'elle est extrêmement critique. La trame de l'histoire se déroule en effet sur un pittoresque sarcastique que n'importe quel adolescent comprendra. Tous les personnages de ce roman, de Maria jusqu'au curé, sont présentés par un narrateur critique souvent jusqu'à l'humour, mais jamais jusqu'au mépris. Par ailleurs, cette histoire, qui est racontée de manière critique, a elle-même une fonction critique dans l'univers romanesque: I'histoire d'amour de Maria est celle qui ne pouvait se produire qu'au «Pays de Québec" où la situe le roman. Louis Hémon ne raconte-t-il pas à peu près la même "histoire» que Flaubert dans Madame Bovary? II en aura simplement déplacé les chapitres: Maria est amoureuse de Léon qui disparaît bientôt et elle doit choisir entre Charles et Rodolphe. Mais pas de coup de théâtre, rien d'emporté et surtout pas d'arsenic: elle choisit Charles en connaissance de cause. Bref, il suffit d'imaginer un instant que Louis Hémon réécrit Madame Bovary selon la recette éprouvée de Patrice Lacombe ("Laissons aux vieux pays, que la civilisation a gâtés, leurs romans ensanglantés, peignons l'enfant du sol, tel qu'il est, religieux, honnête, paisible de mcurs, etc. "), pour comprendre quelle foudroyante ironie il y avait à raconter cette histoire beaucoup trop aimable, belle et douce pour être simplement vraisemblable. Un enfant de chœur ou une dame de sainte Anne n'auraient jamais pu imaginer rien de plus attendrissant. Telle quelle, cette histoire est en effet inimaginable ailleurs qu'au "Pays de Québec" tel qu'il se trouve ainsi impitoyablement imaginé ou caractérisé dans ce roman. Voilà en tout cas de quoi exercer le sens critique d'un jeune lecteur. Et il en est 
de même encore de toute la perspective poétique et symbolique du roman qui joue toujours sur deux tableaux: de la carte postale à l'eau de rose et de l'ironie de cette poésie facile. Bref, il s'agit d'un roman pour adolescents particulièrement bien réussi, ni bête, ni mensonger, ni moralisateur. Mais, bien entendu, cela reste un roman pour adolescents, dont certains passages comme celui-ci sont tout simplement enfantins: "Le dernier maringouin vint se poser sur la figure de la petite Alma-Rose. Gravement elle récita les paroles sacramentelles: «Mouche, mouche diabolique! Mon nez n'est pas une place publique!» Puis elle écrasa prestement la bestiole d'une tape.» (pp. 61-62)

\section{Le chef-d'cuvre était un canular}

S'il existe un mythe de Maria Chapdelaine, s'il y a une critique tout à fait délirante de ce roman, c'est tout simplement parce qu'on n'a pas vu qu'il s'agissait d'un roman pour adolescents qui n'avait rien à voir avec l'«immortel chef-d'œuvre" d'une littérature nationale. Or non seulement Louis Hémon a écrit un roman pour adolescents, mais il n'a jamais voulu écrire autre chose, comme le montre l'histoire littéraire. Maria Chapdelaine ne se détache pas de la littérature française où, à l'époque de Marcel Proust et de Jacques Rivière, le roman populaire est le néo-réalisme exotique qui situe à l'étranger les ingrédients du fascisme de l'entre-deux guerres, le nationalisme et le chauvinisme français. Le roman de Louis Hémon en est une réalisation parfaite sur le modèle du recueil de contes d'André Savignon, les Filles de la Pluie (publié par Grasset), qui se mérite le prix Goncourt en 1912. Or du Lac-Saint-Jean, Louis Hémon en demande justement un exemplaire à sa mère le 7 janvier 1913 et à cette occasion il le décrit précisément par son caractère exotique, puisqu'il s'agit, comme le dit son sous-titre, de "scènes de la vie ouessantine»: "Veux-tu avoir la bonté de m'envoyer le bouquin sur Ouessant: Les filles de la pluie." (Correspondance, p. 187). À ce moment, Louis Hémon a déjà commencé à écrire Maria Chapdelaine; il l'aura probablement écrit et aura peut-être commencé à le mettre au net lorsqu'il recevra enfin le livre de Savignon le 25 avril. Par conséquent, ce n'est pas le contenu du recueil de Savignon qui importe, mais bien le genre ou la perspective littéraire qu'il représente.

Or pour évaluer le genre littéraire de Maria Chapdelaine, il faut non seulement rapprocher ce roman des Filles de la pluie, mais également l'opposer aux autres romans dont Louis Hémon est l'auteur. Cela est particulièrement net à la lecture de Monsieur Ripois et la Némésis (publié par Grasset en 1950). II suffit de lire cette histoire d'un don Juan cynique et particulièrement immoral (mais qui entendra lui aussi des voix réparatrices à la fin du roman) pour comprendre que Maria Chapdelaine a très précisément la valeur d'un canular. Voici ce que donnerait dans ce roman l'épisode de Maria Chapdelaine aux bleuets avec... monsieur Ripois:

Mabel finit pourtant par se dégager au bout de quelques instants, mais ce fut pour dire: 
- Vous ne devez pas être très confortable, cher! Asseyez-vous dans le fauteuil, si vous voulez, je m'assiérai sur vos genoux.

«Cette fois, se dit M. Ripois, je crois que ça y est!» Et, quand il la sentit presque couchée sur lui, la tête sur son épaule, il recommença à l'embrasser ardemment en lui caressant les seins. A mesure que ces caresses devenaient plus hardies, il cherchait à deviner d'avance comment elles seraient reçues; car il avait beau se répéter qu'une femme qui s'abandonne ainsi est prête à s'abandonner tout entière, une certaine hésitation le retenait malgré tout. Savait-on jamais, avec ces Anglaises! (pp. 77-78).

Et la description se fait ainsi de plus en plus précise jusqu'à ce que monsieur Ripois risque "un geste plus précis". Voilà qui nous change un peu des tendres regards de François Paradis.

On ne sait pourquoi, Louis Hémon s'est fait venir au Québec une copie de son manuscrit de Monsieur Ripois et la Némésis qu'il reçoit en même temps que les Filles de la pluie. Sa sceur Marie lui demande alors s'il compte faire paraître ce roman au Canada, ce à quoi Hémon répond: «Non! II ne paraîtra pas ici! Si tu connaissais le Canada français, tu n'émettrais pas de supposition aussi comique.» (Correspondance, pp. 196-197). Cette hypothèse est en effet des plus drôles, surtout lorsque l'on sait que Grasset cachera durant plus de vingt-cinq ans que l'auteur de Maria Chapdelaine était aussi l'auteur de ce roman. Mais le plus comique est bien plutôt l'inverse. C'est-à-dire que l'auteur de Monsieur Ripois et la Némésis écrira Maria Chapdelaine, ce roman pudibond, tendre et charmant, et qu'il l'écrira pour rendre compte du Canada français des années 1910. Dès lors, on peut être absolument certain que non seulement Louis Hémon a écrit un roman pour adolescents. mais que c'est très précisément ce qu'il a voulu faire et, par-dessus le marché, ce qu'il a parfaitement bien réussi. II ne destine certes pas son roman à la «Bibliothèque verte" ou à la "Bibliothèque de la jeunesse» de la maison Hachette, où des centaines de milliers d'exemplaires en seront vendus plus tard, mais cette destinée est à elle seule une excellente description de la structure et des contenus du genre "roman pour adolescents" dont relève manifestement Maria Chapdelaine (on mettra sans peine le roman à sa place et dans l'histoire et dans les catégories et marques du genre à l'aide de la synthèse d'André Bay, "La littérature enfantine», dans I'Histoire des littératures, Encyclopédie de la Pléiade, vol. III, p. 1604-1621). La première et la plus importante ironie critique de Maria Chapdelaine est donc simplement d'être (objectivement et absolument) un roman pour adolescents qui se présente comme un « récit du Canada français». Avec un petit coup de pouce, le récit devient un chef-d'œuvre et, par conséquent, un incroyable canular.

\section{Un livre critique réussi jusque dans son style}

Louis Hémon n'est pas, bien entendu, responsable de la mystification, bien que ce soit le danger qui guette toujours l'ironie critique: elle risque d'être prise au sérieux par les imbéciles. Ce qui n'est pas non plus sans charmes. Voilà en effet le côté grand guignol du mythe de Maria Chapdelaine, tout le 
cirque de l'Aventure Louis Hémon (Fides, 1974) racontée par Alfred Ayotte et Victor Tremblay d'une manière qu'il est impossible de lire sans rire. Pourtant l'ironie critique du narrateur était évidente du début à la fin de son roman pour adolescents et à tous les niveaux, depuis la caricature des personnages typiques jusqu'aux jugements sur ce Pays de Québec. II suffisait de voir comment s'y trouvait décrite la religion des personnages (dévotion populaire, contenu théologique et fonction sociale) pour savoir que le narrateur du roman n'était pas à genoux avec ses personnages...

S'il y avait encore des incrédules avec la tête aussi dure que croche, il suffirait aujourd'hui de les envoyer lire la correspondance de Louis Hémon avec sa famille. Elle a été éditée par Nicole Deschamps aux Presses de I'Université de Montréal en 1968 et rééditée au Boréal Express en 1980. On y verra en particulier quelle grossière erreur d'interprétation les éditeurs de Maria Chapdelaine ont faite en plaçant entre guillemets ou en italique des mots de la langue parlée au Québec. Non seulement Louis Hémon ne les avait soulignés d'aucune manière dans son roman, mais il écrivait au contraire à sa mère (et justement entre guillemets) le 28 octobre 1911: «Je commence à parler canadien comme un indigène. Je prends les "chars" (tramways électriques), je parle tout naturellement de la «chambre de bains" et de la «chambre à dîner" sur le même "plancher» (étage), etc... C'est une langue bien curieuse» (Correspondance, p. 159). À travers la curiosité, la sympathie, la moquerie et l'ironie de Louis Hémon dans cette correspondance et dans cet extrait, on voit bien l'importance stylistique qu'aura la diction, le vocabulaire, la morphologie et la syntaxe du français tel qu'il se parle au Québec dans son roman. En effet, Maria Chapde/aine n'est pas un roman écrit selon les normes du français international qui ne s'est jamais parlé ailleurs qu'à la radio (il faut y ajouter aujourd'hui la télévision), mais qui s'écrit à peu près partout depuis toujours. Bien au contraire, comme l'ont fait plusieurs romanciers québécois depuis quelques décennies (et comme le font au moins depuis Rabelais au XVle siècle les romanciers français), Louis Hémon a inventé pour son roman une langue ou du moins un style à partir de la langue française telle qu'il l'entendait parler au Québec, avec ses tournures populaires, ses régionalismes et ses anglicismes. Or les éditeurs de Maria Chapdelaine ont brouillé ce style ou cette langue littéraire en plaçant un peu partout et au hasard des mots entre guillemets, ce que l'auteur n'avait jamais fait et ce qui était complètement absurde dans la mesure où ce n'étaient pas le vocabulaire, le lexique ou les mots qui étaient différents de ceux du français international, mais bien toute la langue de ce roman, depuis la diction jusqu'à la syntaxe. À travers cette langue littéraire, toute l'histoire de Maria Chapdelaine devient «indigène» et «curieuse». On voit donc quel impact considérable il y avait à raconter le plus simplement et le plus naturellement possible (c'est-à-dire sans guillemets) dans cette langue cette histoire pour adolescents... à des adultes! On voit du même coup l'importance de l'édition critique de Maria Chapdelaine par Ghislaine Legendre, puisqu'il s'agit de la première édition conforme au manuscrit de Louis Hémon. 


\section{2 - LA PREMIÉRE ÉDITION INTÉGRALE}

On a vu que Maria Chapdelaine était un excellent petit livre pour adolescents déguisé en chef-d'œuvre de la littérature nationale du Québec. Dans ces conditions, on comprend que ce roman, avec tous les événements dont on a su l'entourer (le dernier était le centenaire de la naissance de Louis Hémon), ait donné lieu à un très grand nombre de comptes rendus, d'études et d'éditions de toutes sortes. La bibliographie du Mythe de Maria Chapdelaine de Nicole Deschamps, Raymonde Héroux et Normand Villeneuve fait à elle seule trente pages. Or le texte du roman n'a jamais été publié que sur la base d'une édition journalistique.

\section{Les éditions du roman}

En effet, Maria Chapdelaine est parue après la mort de Louis Hémon en feuilleton du 27 janvier au 19 février 1914, dans le journal français Le Temps (c'est aujourd'hui Le Monde). L'auteur avait fait parvenir à ce journal une des deux copies au carbone de son manuscrit. En même temps, il s'était posté à l'adresse de sa famille à Paris l'original et la première copie au carbone de son manuscrit dactylographié de 171 pages. Ce manuscrit et cette copie, qui étaient la propriété de Lydia Kathleen Hémon, fille de Louis Hémon, se trouvent depuis 1966 à la Bibliothèque de I'Université de Montréal. Or ce manuscrit a fait l'objet pour la première fois en 1980 d'une édition critique par Ghislaine Legendre aux Éditions du Boréal Express. II s'agit d'un établissement rigoureusement scientifique de Maria Chapdelaine qui profite de la confrontation des onze éditions des quatre premiers éditeurs littéraires du roman. Après l'édition journalistique du Temps (1914) qui leur sert toujours de texte de base, il s'agit des éditions de J.-A. LeFebvre (1916), Bernard Grasset (deux en 1921, puis 1924, 1961 et 1967) et Fides (1946, 1957, 1960 et 1970). A la fin du livre de Ghislaine Legendre, on trouve une liste complète des variantes de ces onze éditions que Nicole Deschamps étudie dans son Avant-propos. Nicole Deschamps dit à ce propos qu'il s'agit d'un relevé des «principales variantes». Le spécialiste, qui sera bien le seul à s'intéresser de près à ces variantes, aura compris qu'il s'agit des variantes significatives (c'est-à-dire qui ont un sens sans qu'il soit nécessaire de retourner au manuscrit pour les comprendre: il est bien évident qu'on ne va pas faire payer à cinq ou dix mille lecteurs le coût du relevé de quelque mille variantes de ponctuation qu'aucun spécialiste ne pourra jamais étudier sans se reporter au manuscrit). Ce relevé ne comprend donc pas, bien entendu, les erreurs de frappe ou les fautes d'orthographe du manuscrit, ni les variantes de la ponctuation, ni non plus les coquilles des éditions dépouillées. Par contre, les corrections de Louis Hémon dans son manuscrit sont soigneusement relevées.

Cette édition scientifique constitue donc un événement littéraire majeur de ces dernières années, non seulement dans le monde de l'édition québécoise, mais également pour l'histoire des littératures d'expression française, étant donné l'éclat mythique du roman en question. 


\section{Les manipulations du texte du roman}

II n'est pas nécessaire d'être un spécialiste de l'édition critique pour comprendre l'importance de l'établissement d'un texte littéraire. Avant de l'étudier, il faut s'assurer qu'on puisse le lire dans une édition conforme, c'est-à-dire qui respecte le texte écrit par l'auteur. Or dans le cas de Maria Chapdelaine, les manipulations auxquelles on a soumis le texte de Louis Hémon sont si nombreuses et si importantes que l'édition de Ghislaine Legendre est non seulement la première édition conforme, mais pour bien dire la première édition intégrale du roman.

En effet, toutes les éditions de Maria Chapdelaine sans aucune exception avaient été faites à partir de l'édition journalistique du roman paru en feuilleton dans le journal Le Temps. Cette première édition, même si elle était très soignée, transformait considérablement et de plusieurs manières la lettre du texte de Louis Hémon, particulièrement en regard des normes de l'édition scientifique moderne. En plus des fautes d'édition courantes (coquilles typographiques, fautes de lecture et d'interprétation), le manuscrit de Louis Hémon était soumis à toute une série de corrections lexicales, morphologiques, syntaxiques et stylistiques, sans oublier encore la normalisation typographique (ponctuation, usage des majuscules et divisions en paragraphes sont souvent transformés). Ainsi, une racine (race) ancienne avait retrouvé son adolescence sur un plan (pan) du continent nouveau où les familles canadiennes (canayennes) travaillent tout l'après-midi (l'avantmidi) avant le dîner où l'on mange bien entendu des atocas (atacas). C'est de malheur (valeur), mais il a même fallu ajouter de beaux gros seins à Maria Chapdelaine pour lui faire une poitrine forte (profonde) alors que Louis Hémon lui avait dessiné, avec sa poésie jusque-là symbolique, un giron... (image qui lui vient probablement d'Homère, "les Dardaniennes au sein profond", lliade, $18: 340$ ).

Mais tout cela, c'est encore des pinottes à côté de la manipulation la plus importante, la plus désastreuse et la plus significative qui a consisté à mettre entre guillemets et en italique des éléments de la prononciation et du lexique de Maria Chapdelaine. Ainsi des mots comme "foreman», «icitte», "cash", "écureux», "cent» ou "adon», c'est-à-dire des mots choisis au hasard et dont la seule caractéristique commune était de n'être pas du français international, sont mis de cette manière en relief et à l'écart. On a déjà vu quelle importance considérable avait le fait que Louis Hémon ne les ait marqués d'aucune manière. II n'est pas difficile de comprendre que cette manipulation transformait chacune des pages du roman et qu'elle en dégradait radicalement le langage littéraire.

Comme si cela n'était pas suffisant, les trois éditeurs suivants vont encore apporter à cette édition journalistique encore d'autres erreurs et encore d'autres corrections. Et comme c'est à Montréal que nous pairlons le mieux françâ, ce sont bien entendu les éditions Lefebvre, puis Fides qui vont apporter au roman les plus belles corrections: au lieu qu'il fasse ben fret, il va faire... "bien fret»! 


\section{La première édition conforme et intégrale}

Dans ces conditions, on comprend quelle importance prend l'édition critique de Maria Chapdelaine par Ghislaine Legendre, puisqu'elle permet pour la première fois d'en venir au texte réellement écrit par Louis Hémon, au lieu de lire une «mise entre guillemets de la réalité québécoise», comme l'écrit si justement Nicole Deschamps dans son avant-propos. Mais l'importance considérable de cette édition critique ne s'arrête pas là. Elle tient encore à la qualité même de l'édition.

D'abord, la qualité du travail des Éditions du Boréal Express doit être signalée. Ce livre est d'une parfaite sobriété dans sa présentation, sa mise en page et sa composition, laissant tout l'espace et toute la vedette au texte de Louis Hémon. C'est d'ailleurs lui seul qui est annoncé sur la couverture. Sobriété aussi de la publicité, quand on pense à la page titre qu'on aurait pu en faire à grand renfort d' "édition critique" par un «professeur de l'Université de Montréal", etc. Au lieu de cela, l'éditeur annonce simplement la première édition intégrale et la première édition conforme au manuscrit de Louis Hémon, ce qui est rigoureusement exact. La dernière, mais la plus importante réussite du Boréal Express, est le prix de son livre: 8,95 \$. Il ne reste qu'à en faire une réussite commerciale, ce qui me paraît assuré.

Ensuite, la qualité du travail de Ghislaine Legendre me paraît d'autant plus remarquable qu'elle est toute entière au service de Maria Chapdelaine et de ses lecteurs. Pourtant, rien n'aurait été plus facile pour Ghislaine Legendre que de nous réaliser une de ces belles "éditions critiques", une édition aussi savante qu'inutile, dans tous les cas hors de prix (sans compter le coût en subventions fédérales et provinciales), et qui n'aurait été "consultée" que par trois «spécialistes» comme Aurélien Boivin, Raymond Paul et Réal Ouellet qui se seraient empressés d'en faire un compte rendu élogieux, trop heureux d'être les trois seuls savants capables de s'y retrouver dans l'«instrument de bibliothèque" qui aurait alors été digne de toute notre admiration. Au lieu de toute cette suffisance universitaire, Ghislaine Legendre s'est tout simplement mise au service des lecteurs du Québec. Et elle l'a fait avec une compétence et une sobriété dont son éditeur s'est rendu digne.

En effet, son travail est tout entier au service du texte qu'elle a édité. L'apparat critique, c'est-à-dire les notes sur le texte, la liste et la description des variantes et l'histoire du texte, bref tout ce qui explique et justifie l'établissement scientifique d'un texte, cet apparat critique donc tient en moins de vingt pages d'un livre qui en compte deux cent trente. On trouve à la fin du livre un relevé des variantes et une liste des notes de huit pages, puis un index des personnages et des lieux de quatre pages. Par ailleurs, le livre s'ouvre sur un avant-propos de Nicole Deschamps en sept pages. Le lecteur simplement intéressé au texte de Louis Hémon lira Maria Chapdelaine dans une édition absolument irréprochable, sans être distrait d'aucune manière par cet "apparat critique" et sans même savoir qu'il s'agit d'une "édition critique». Au contraire, un spécialiste (mettons un professeur de littérature) comprendra tout de suite que Ghislaine Legendre, qui a déjà 
édité les Annales de Marie Morin aux Presses de l'Université de Montréal, est responsable de l'édition critique, puisqu'elle en signe les notes et les variantes. Par contre, Nicole Deschamps s'est chargée, dans l'avant-propos, de présenter les quatre états qui marquent la filiation des onze éditions de Maria Chapdelaine à partir desquelles toutes les autres ont été produites. Je crois qu'on ne saurait trop insister sur l'extraordinaire sobriété, la modestie et la discrétion de toute cette science entièrement au service du texte de Louis Hémon.

Pour nos enfants, nous avons Passe-Partout, une émission de télévision que nous pouvons être fiers de leur offrir. Pour nos jeunes adolescents, nous avons maintenant une édition de Maria Chapdelaine, un roman parfaitement bien adapté à leur âge, que nous pourrons aussi être fiers de leur offrir, non sans prendre d'abord le plaisir de le lire nous-mêmes pour la première fois tel que l'avait écrit Louis Hémon.

\section{Un acte politique}

A cette occasion, on pourra réfléchir au contenu politique de Maria Chapdelaine que l'édition de Ghislaine Legendre met brutalement en évidence. Car la publication de cette édition critique est tout autant un acte politique qu'un acte scientifique. Le contenu politique du roman est à la fois simple et vague, à l'image de l'histoire d'amour qui s'y trouve racontée. Mais Louis Hémon lui a donné beaucoup d'importance en faisant de cette observation politique le moteur de la décision que prend Maria de rester au Québec à la fin du roman. On n'a pas à se demander si cette motivation est vraisemblable, pas plus qu'on peut se demander s'il est vraisemblable que Maria pense abandonner sa famille pour suivre Ephrem Surprenant aux États-Unis, car ce n'est pas nous qui écrivons ce roman, mais Louis Hémon qui fait vivre à Maria le dilemme cornélien de savoir si elle suivra Ephrem ou si elle restera, ce qui signifie dans ce cas qu'elle épousera Eutrope Gagnon, son voisin. Or la voix intérieure qui lui «commande» de rester et à laquelle elle se «soumet» ("Le souvenir de ses autres devoirs ne vint qu'ensuite") est une voix politique. La première voix parle de la géographie du pays et la seconde de sa culture, de sa langue surtout, mais la troisième, la plus importante, est "la voix du pays de Québec». Or cette voix va répondre à la question posée par Maria (manifestement pour le narrateur) de la manière suivante: "Tout de même... c'est un pays dur, icitte. Pourquoi rester?». À travers la rêverie du narrateur français (qui parle de la «splendeur» et de "la force barbare du pays neuf où une race ancienne a retrouvé son adolescence»l, la réponse constitue une observation politique toute simple encore très juste aujourd'hui: "Autour de nous des étrangers sont venus, qu'il nous plaît d'appeler des barbares; ils ont pris presque tout le pouvoir; ils ont acquis presque tout l'argent; mais au pays de Québec rien n'a changé. Rien ne changera, parce que nous sommes un témoignage." Voilà qui est précis et concret, mais également bien vague et poétique. D'une part la réalité, un petit peuple francophone exploité, dominé, colonisé par le monde anglo-saxon qui l'étouffe plus qu'il ne l'entoure, de l'autre cette motivation aussi abstraite que farfelue de vouloir être et rester 
un témoignage, quand il s'agit tout simplement de vivre, d'être chez soi (la première voix) et d'être soi-même, ce qu'il est bien difficile d'être autrement qu'en français au Québec (la deuxième voix). Et voilà pourquoi ma fille est muette et que Maria n'est pas partie pour les États, ce qui ne porte bien entendu pas à conséquence dans un roman pour adolescents. Pourtant, voilà justement ce qui explique le mythe de Maria Chapde/aine. Car force est de constater qu' "autour de nous des étrangers sont venus" et qu'ils y sont encore, qu'«ils ont pris presque tout le pouvoir» et qu'ils l'ont encore et qu'«ils ont acquis presque tout l'argent» et qu'ils l'ont encore, de sorte qu'«au pays de Québec rien n'a changé». Or cette situation explique le mythe de Maria Chapdelaine: c'est parce que nous sommes économiquement et politiquement dominés par le monde anglo-saxon américain que nous avons pu être colonisés culturellement et intellectuellement avec le roman de Louis Hémon, mis entre guillemets par la droite catholique, apostolique et fasciste française de l'entre-deux guerres - et mis en apostrophe aujourd'hui.

En forçant un peu les faits, admettons qu'il n'y ait pas que les financiers qui soient aujourd'hui fédéralistes et que les intellectuels qui soient indépendantistes, il reste que le Canada peut se développer (rapatrier sa Constitution, par exemple) comme si le Québec n'existait pas; et que la voix de celui-ci est encore "à moitié un chant de femme et à moitié un sermon de prêtre». Quoi qu'on rêve pour l'avenir, le pays de Québec reste encore un "pan de continent", cet espace sans frontières qui se perd dans l'Amérique où vivent des barbares, tout le contraire des voisins qu'ont tous les pays du monde. Bref, nous n'avons toujours pas réalisé l'indépendance du Québec, question que Louis Hémon n'a pas posée autrement qu'en observant que nous étions soumis à des étrangers. II l'a fait en quatre lignes, au centre d'une réflexion socio-culturelle bien "poétique» destinée à motiver la décision de Maria qui, de toutes façons, ne fait aucun doute.

Par ce détour, je voulais dire qu'il n'était ni possible ni souhaitable d'effacer le mythe de Maria Chapdelaine, mais qu'il était grand temps qu'on lise tel quel un roman qui justement aide à l'expliquer, en plus d'en avoir été... l'objet! Dans ce petit roman gentiment sarcastique, Louis Hémon nous a largement vus tel que nous sommes, bien capables par exemple de faire un chef-d'œuvre d'un roman pour adolescents. On s'en consolera en se disant qu'on n'a pas été les seuls à se faire prendre à cet extraordinaire canular. Si Nicole Deschamps avait crevé le mythe en 1968 en éditant la correspondance de Louis Hémon avec sa famille et si elle en a fait avec Raymonde Héroux et Normand Villeneuve une histoire tout à la fois tendre et humoristique, c'est à Ghislaine Legendre qu'il est revenu de le dégonfler complètement. Elle l'a fait en nous ramenant très exactement où il avait commencé: au roman de Louis Hémon. Car s'il y avait des raisons politiques de manipuler ce roman, et dans son texte et dans son sens, la publication intégrale du roman est du même coup un acte politique. Dès lors, on peut se demander pourquoi un travail si simple, si bien fait et si important a été si mal accueilli des spécialistes. 


\section{3 - LES COMPTES RENDUS DES SPÉCIALISTES}

Le mythe de Maria Chapdelaine est si peu surprenant au Québec que nos spécialistes viennent de nous créer le mythe de l'«édition critique» pour rejeter l'édition de Ghislaine Legendre qui l'anéantit. L'édition de Maria Chapdelaine par Ghislaine Legendre serait en effet entachée de rien de moins que de manque de "professionnalisme" et on attendrait toujours l'«édition critique définitive» du roman. Déconcerté par l'incroyable compte rendu de cette édition par Réal Ouellet dans la revue Lettres québécoises, j'ai consulté le dossier Louis Hémon du Centre de littérature québécoise de l'Université de Montréal (au département d'Études françaises où je suis professeur) et j'y ai trouvé cinq comptes rendus de ce livre. D'abord la chronique de Jean Royer parue dans Le Devoir (18 octobre 1980, pp. 21 et 40) et ensuite le compte rendu d'André Bourin paru dans la Revue des Deux Mondes (mai 1981. pp. 439-443). Ils disent quelques mots seulement du travail d'édition de Ghislaine Legendre à l'occasion de la publication du Mythe de Maria Chapdelaine dont ils rendent compte tous deux avec une parfaite compétence. Les trois autres comptes rendus sont un peu plus surprenants. II s'agit de trois spécialistes qui non seulement font un compte rendu défavorable de l'édition de Ghislaine Legendre, mais tombent à bras raccourcis sur Nicole Deschamps qui en a signé, on le sait, l'avant-propos. Cela me paraît très grave dans la mesure où il s'agit pour ces spécialistes de l'édition d'informer d'autres spécialistes sur l'une des quelques rares éditions critiques qui paraissent au Québec chaque année. Un étranger qui s'intéresse à la littérature québécoise ne va probablement pas dépouiller Le Devoir. Il consultera la bibliographie courante annuelle Livres et Auteurs québécois. S'il veut cerner de plus près la production littéraire québécoise, il consultera la revue Lettres québécoises qui paraît quatre fois par année. Par contre, s'il s'agit d'un professeur de français langue seconde ou d'un professeur de littérature canadien ou américain, il lira probablement aussi Québec français. Or c'est dans ces trois revues que sont parus respectivement les comptes rendus de Raymond Paul, Réal Ouellet et Aurélien Boivin. Ces comptes rendus ne vont donc pas seulement causer durant plusieurs années un tort irréparable au travail réalisé par Ghislaine Legendre, nuire aux chercheurs étrangers et aux chercheurs à venir, mais vont surtout discréditer injustement les chercheurs québécois qu'ils représentent en les ravalant au niveau de leur compte rendu. Bien entendu, aucun mauvais livre ne doit être épargné et la critique doit toujours être absolument impitoyable. Mais il me semble qu'on peut difficilement cacher les qualités ou l'importance d'un livre dont on prétend faire le compte rendu - à moins, bien sûr, de ne pas les voir, ce qui serait de l'incompétence dans un domaine aussi concret que l'édition critique. Or dans ces trois comptes rendus, la faiblesse et la pauvreté des arguments critiques étonnent tout autant que la virulence des propos qui ne parviennent même pas à s'y appuyer. Tout se passe comme si ces comptes rendus n'étaient que de simples règlements de compte qu'on n'aurait même pas pris la peine de maquiller. 


\section{Livres et Auteurs québécois}

Le compte rendu le plus virulent est celui qu'a fait paraître Raymond Paul, professeur au cégep Édouard-Montpetit, dans Livres et Auteurs québécois (1980, pp. 44-45). Pourtant, le compte rendu le plus virulent paru dans la revue la plus importante est en même temps le plus inoffensif. N'importe quel lecteur devrait comprendre qu'il est entièrement dicté par la frustration. Raymond Paul nous dit en effet à la dernière ligne de son compte rendu qu'il est bien fâché de ne pas avoir été cité par Nicole Deschamps dans son avant-propos, ce qu'il complète malheureusement d'une accusation voilée extrêmement grave qui met en cause l'honnêteté intellectuelle de Nicole Deschamps. En parlant de sa thèse sur "L'établissement du texte de Maria Chapdelaine" qu'il a faite sous la direction de Nicole Deschamps, il s'étonne que celle-ci n'ait pas cité son travail "qu'elle a très certainement consulté, voire même utilisé" ".

Je me suis donc rendu à la Bibliothèque des Sciences sociales de I'Université de Montréal pour consulter son travail. Il s'agit de l'ouvrage suivant: Établissement du texte de "Maria Chapdelaine», Mémoire de Maîtrise ès-Arts, Études françaises, Université de Montréal, août 1973, 205 pages. D'abord, il ne s'agit pas d'une thèse, mais d'un mémoire de Maîtrise. Ensuite, il ne s'agit pas d'un établissement du texte, comme le dit le titre, mais d'un simple relevé des variantes de trois éditions seulement (une de chacun des éditeurs) et du manuscrit. L'étudiant (qu'était alors le professeur Raymond Paul) a fait un travail de dépouillement, de confrontation et de relevé considérable, un travail qui lui a valu le diplôme de Maîtrise. Ce travail, toutefois, est tout simplement inutilisable. D'une part parce que le texte de base qu'il a choisi pour cataloguer ses relevés est l'édition journalistique du roman paru en feuilleton dans le Temps, et d'autre part parce qu'il a relevé systématiquement et sans aucune analyse tout ce qui lui passait sous les yeux, ce qui donne un relevé aussi lourd qu'inutile et inutilisable. Sans compter le relevé systématique des variantes de ponctuation et de graphie, Raymond Paul nous inflige toutes les fautes de frappe de Louis Hémon. Or Dieu sait qu'il ne tapait pas très bien à la machine à écrire. On apprend donc à chaque page que Louis Hémon a, sous la lettre $c$, ajouté la cédille au crayon ou qu'il a tapé un i sous un e dans l'article «le», ce qui est franchement à mourir de rire. Bref, il s'agit d'un travail de Bénédictin, mais d'un Bénédictin un peu jeune et pas très doué.

Même dans le cas d'un travail de Maîtrise, on aurait attendu après un tel relevé une analyse un peu consistante des variantes relevées. Cette analyse tient en exactement quinze pages, soit son introduction. Certes, Raymond Paul a bien vu et bien présenté les principales transformations du texte de l'une à l'autre des trois éditions et quelques-unes de ses conclusions (notamment pages 13 et 14 qui décrivent bien notre situation culturelle dont témoigne l'édition de J.-A. Lefebvre) se rapprochent de celles qu'on trouve dans l'avant-propos de Nicole Deschamps; mais cela est attendu dans la mesure où il est évident pour tout le monde que l'édition du Temps modifie la disposition des paragraphes ou divise les phrases trop longues du manuscrit; dans la 
mesure aussi où il est évident (mais pas pour tout le monde) que les manipulations de l'édition de J.-A. Lefebvre sont significatives de notre situation culturelle. Mais là s'arrête la comparaison. Il suffit en effet de lire la première phrase de son travail pour comprendre que les conclusions de Raymond Paul sont contradictoires par rapport à celles de Nicole Deschamps: "Le succès international de Maria Chapdelaine dévoila l'existence d'une vie culturelle française en Amérique du Nord.» Du même coup, on se rend compte que c'est exactement l'inverse qui est vrai: les quelques conclusions intéressantes de son introduction lui ont été de toute évidence inspirées par Nicole Deschamps, la directrice de son travail. Ces idées encore informes, mal intégrées à son introduction et mal exprimées sont bien entendu ce qu'un étudiant a réussi à comprendre de ce que lui expliquait Nicole Deschamps. Celle-ci n'a tout de même pas réussi à exprimer mieux que Raymond Paul... les idées de Raymond Paul!

Avec tout cela, il faut dire que ce n'était pas la place dans un avant-propos (où absolument aucune étude n'est citée) de renvoyer à un mémoire de Maîtrise qui n'a d'aucune manière été utilisé dans l'édition de Ghislaine Legendre sur laquelle s'appuie exclusivement Nicole Deschamps. Par contre, celle-ci l'a très correctement répertorié dans le Mythe de Maria Chapdelaine et l'a cité dès la première page de son livre. C'était à mon avis lui faire beaucoup trop d'honneur (voyez sa belle reconnaissancel). Bien entendu, ce mémoire de Maîtrise ne peut pas être jugé comme s'il s'agissait d'un travail qui aurait donné lieu à une publication et il est évident qu'il ne méritait pas que je dise ce que j'en pense. Mais le professeur Raymond Paul devait savoir qu'en se citant en exemple pour déprécier le travail d'un autre, il s'exposait à ce que quelqu'un aille voir ce qu'il avait fait à l'époque où il était étudiant. Pourtant, je l'épargnerai en ne parlant pas de son style: chacun peut le juger à la lecture de son compte rendu.

Pour finir, les critiques que formule Raymond Paul à l'édition de Ghislaine Legendre prouvent qu'il n'a pas continué de réfléchir aux problèmes de l'édition critique depuis la rédaction de son mémoire. II ne s'est même pas intéressé aux éditions qui ont été dépouillées. II se demande quelle méthode Ghislaine Legendre a utilisée pour retenir les variantes (comme si ce n'était pas le bon sens qui le disait) et il constate que les fautes d'orthographe et la ponctuation fautive du manuscrit ont été corrigées: il aurait voulu lire un exposé en bonne et due forme là-dessus. II pousse de hauts cris, comme les deux autres spécialistes, à propos des majuscules que Ghislaine Legendre n'a pas laissées aux noms de mois et de points cardinaux. Enfin, comme tous les spécialiștes, il aurait bien voulu voir des petits numéros partout dans le texte de Maria Chapdelaine, au lieu que les variantes soient simplement énumérées à la fin du livre pour les spécialistes comme lui que cela intéresse. Rien de tout cela, on le voit, ne met en cause l'édition critique dont Raymond Paul n'a tout simplement pas rendu compte. II s'est contenté d'en brandir le mythe. 


\section{Québec français}

Aurélien Boivin, lui, travaille chez Fides. II n'a donc pas aimé que Ghislaine Legendre nous prouve hors de tout doute que les éditions de Maria Chapdelaine par cette maison d'édition étaient les plus fautives. II ne s'agit pas de ma part d'un procès d'intention, puisqu'Aurélien Boivin nous le dit lui-même au cours de son compte rendu dans le Québec français (décembre 1980, pp. 62-64): «la maison Fides, contre qui madame Deschamps semble avoir bien de la hargne!», écrit-il entre parenthèses. Or Nicole Deschamps n'a jamais fait preuve de la moindre hargne contre la maison Fides, puisqu'elle a eu au contraire le tact de ne pas en dire un seul mot dans tout son avant-propos. Pas un mot. C'est bien plutôt Ghislaine Legendre, en quelque sorte malgré elle, qui a fait froidement la liste des fautes des éditions de Maria Chapdelaine par la maison Fides dans son relevé des variantes.

II est vrai aussi que l'édition de Ghislaine Legendre discrédite et périme complètement les éditions du roman par Fides, dont (c'est bien de malheur!) celles parues la même année, une nouvelle édition de la collection "Nénuphar" et surtout celle de la collection «Bibliothèque québécoise " à laquelle Aurélien Boivin a justement collaboré. Je n'ai pas fait de longues recherches pour apprendre tout cela, puisque c'est Aurélien Boivin lui-même qui nous le dit dans son compte rendu où il énumère les éditions de Maria Chapdelaine parues en 1980 . A cette occasion, il propose modestement son édition aux $\approx$ bourses moins garnies" qui ne peuvent pas se payer les belles éditions illustrées dont le coût varie entre 75 et 750 dollars. II ne se rend pas compte que son livre "à prix populaire", malgré la qualité de la préface de Pierre Pagé, est tout simplement un anachronisme depuis la parution de l'édition intégrale et conforme de Ghislaine Legendre.

Voilà qui est triste, mais explique assez bien que l'édition de Ghislaine Legendre ne soit pas "une véritable édition critique. Car il faut le dire aux profanes: l'édition du Boréal Express est, en ce sens, fort décevante.» Les "profanes", ce sont bien entendu les pauvres lecteurs auxquels Aurélien Boivin destine la dernière mouture de l'édition Fides ęn «Bibliothèque québécoise", au lieu de l'édition du Boréal Express. II trouve donc que cette dernière édition n'est pas «la seule édition intégrale» (on ne lui avait jamais rien «soustrait», notion bien courte de l'intégralité), que ce n'est "pas non plus une édition critique» et que "les responsables de cette édition, tout comme l'éditeur d'ailleurs, ne font guère preuve de professionnalisme", sans parler du « manque d'uniformité flagrant de l'édition du Boréal». Bref, l'édition de Ghislaine Legendre est complètement pourrie, d'une médiocrité absolue et appuyée par un battage publicitaire mensonger dans la mesure où les transformations du texte de Maria Chapdelaine par les éditeurs sont tout simplement négligeables. Le bouquet: "Et je ne vois pas comment le fait de reproduire entre guillemets et en italique "les canadianismes, archaïsmes et anglicismes» puisse «déformer franchement» le texte», écrit-il.

Or en tout et pour tout, Aurélien Boivin va relever trois coquilles dans le texte, une faute d'orthographe (l'accord du participe passé dans "avait 
laissées échapper") et six variations lexicologiques (M. le curé et Monsieur le curé, à deux endroits différents du texte). Ces fautes sont bien entendu regrettables et devraient être corrigées dès le prochain tirage. Mais qu'un spécialiste discrédite un travail d'édition sur la base de dix coquilles, cela me paraît tout de même un peu exagéré. Sans compter qu'Aurélien Boivin en ajoute: il veut absolument qu'on écrive «Ferdinand Larouche» alors que Louis Hémon a bel et bien écrit «Ferdina Larouche». Il est vrai qu“à son avis les corrections "ont été apportées par Grasset (ou Le Temps) pour améliorer le texte». II faudrait donc savoir si on veut lire le roman écrit par Louis Hémon ou par... Aurélien Boivin!

Car Aurélien Boivin n'a absolument aucune autre amélioration ou correction à suggérer pour que Ghislaine Legendre et le Boréal Express fassent enfin preuve de «professionnalisme». Ah! j'oubliais: il aurait voulu lui aussi des petits numéros partout dans le texte pour rappeler les variantes. Comme cela, le lecteur aurait bien su qu'il s'agissait d'une "édition critique" faite par un "spécialiste». Le seul problème qu'il pose qui pourrait passer pour relever de l'édition critique est celui de savoir ce qu'il faut entendre par les variantes «principales», comme a eu le malheur de l'écrire Nicole Deschamps à la première phase de son avant-propos. Les éditions dépouillées, le type de corrections apportées par Louis Hémon dans son manuscrit, les catégories, la nature, la fonction ou le sens des variantes relevées chez les éditeurs du roman, absolument rien de tout cela n'intéresse Aurélien Boivin. On se demande donc ce qu'il aurait fait d'une "véritable édition critique». Bref, rien dans tout cela, on le voit, ne met en cause l'édition critique dont Aurélien Boivin n'a tout simplement pas rendu compte. II s'est contenté d'en brandir le mythe.

\section{Lettres québécoises}

La plus décevante des trois analyses "spécialisées" consacrées a l'édition critique de Maria Chapdelaine est à coup sûr celle de Réal Ouellet dans la revue Lettres québécoises ( $n^{\circ} 21$, printemps 1981, pp. 43, 45, 46). Elle est surprenante parce qu'elle contredit absolument ce que Réal Ouellet n'a cessé de dire et d'écrire depuis une décennie sur l'édition critique en domaine québécois. Voici à titre d'exemple ce qu'il écrivait en tête du rapport sur la «Situation de l'édition et de la recherche» (Ottawa, 1978, p. 20) repris dans le premier numéro de la Revue d'Histoire littéraire du Québec: "Je le répète, quelle étude linguistique, thématique ou psychanalytique peut être tentée si le réseau lexical a été transformé intentionnellement par un censeur ou involontairement lors d'impressions successives, lesquelles, comme on sait, ajoutent forcément des fautes.» (1979, p. 11). Est-ce que cette phrase ne s'applique pas absolument, rigoureusement et exactement au texte de Maria Chapdelaine? Alors, est-ce bien le même Réal Ouellet qui écrit, pour discréditer l'édition de Ghislaine Legendre, à propos des onze éditions successives de Maria Chapdelaine conduisant à la plus fautive de toutes, celle publiée par Fides: «Au total de nombreuses retouches, mais qui n'entraînent pas une lecture incorrecte de l'ceuvre.» Bien entendu, il y a une explication contextuelle toute simple: la 
première phrase est écrite pour que nos gouvernements subventionnent l'édition et la seconde pour tapocher sur un livre qui, à ma connaissance, a été réalisé sans aucune subvention, ni l'aide d'aucun groupe d'assistants de recherche. Je sais bien que les idées peuvent varier avec les interlocuteurs, mais il est tout de même surprenant de les voir se contredire aussi radicalement.

La tactique (car c'est bien de tactique qu'il s'agit lorsque les «convictions » se contredisent de cette manière), la tactique consiste donc à minimiser l'impact des transformations apportées au texte de Maria Chapdelaine par ses éditeurs successifs et à ne voir aucune des qualités ni l'importance de l'édition de Ghislaine Legendre pour, bon prince, concéder que «Malgré tout, on tirera grand profit de cette nouvelle édition qui rectifie de nombreuses retouches apportées au texte original. Mais l'on attend toujours l'édition critique et définitive de Maria Chapdelaine. » Or il ne s'agit pas de retouches, il ne s'agit pas non plus de nombreuses retouches, il ne s'agit même pas de rectifier de nombreuses retouches: il s'agit de la première édition intégrale et conforme du manuscrit de Louis Hémon, un événement majeur et d'une portée considérable pour l'étude des littératures d'expression française.

Que le responsable de la chronique des rééditions de la revue Lettres québécoises, qu'un spécialiste de l'édition critique des textes de la NouvelleFrance, que le directeur de l'édition de l'œuvre complète de La Hontan trouve que les manipulations du texte de Maria Chapdelaine par ses éditeurs successifs soient insignifiantes et négligeables et qu'il ne voie ni l'importance considérable de l'édition du manuscrit de Louis Hémon, ni les qualités de l'édition de Ghislaine Legendre, voilà qui me paraît inquiétant, invraisemblable et impardonnable. Je reprendrai donc un à un les "problèmes" soulevés par son compte rendu. On verra que rien dans tout cela ne met en cause l'édition critique dont Réal Ouellet n'a tout simplement pas rendu compte. Mais il faudra se demander pourquoi il en a brandi le mythe, ce qui, on le verra, est une question beaucoup plus difficile.

\section{4 - LE MYTHE DE L'ÉDITION CRITIQUE}

Le compte rendu de Réal Ouellet dans la revue Lettres québécoises ( $\mathrm{n}^{\circ}$ 21, printemps 1981, pp. 43-46) s'intitule «Entre l'héroïsme et la stérilité: Maria Chapdelaine, édition préparée par Nicole Deschamps et Ghislaine Legendre.» On aura compris qu'il s'agit de l'édition de Ghislaine Legendre présentée par Nicole Deschamps, même si cette dernière est la seule qui soit nommée avec insistance dans le compte rendu qui suit son analyse du roman, «entre l'héroïsme et la stérilité», en effet! II ne sera pas inutile de revoir un à un les problèmes soulevés par Réal Ouellet, car ils nous conduisent tout droit au mythe de l'édition critique. J'ai déjà dit que Réal Ouellet dans ce compte rendu ne voyait ni l'importance, ni les qualités de cette édition et qu'il lui inventait tout simplement des défauts. Commençons par ceux-ci. 


\section{L'invention des défauts}

D'abord, Réal Ouellet a trouvé «un certain nombre» de coquilles dans l'édition du Boréal Express. II en a trouvé exactement deux que le lecteur rétablira sans peine lui-même: sans soute pour sans doute à la page 34 et à l'étable en rentra pour à l'étable et rentra à la page 103. Aurélien Boivin, plus attentif, mais non moins sévère, en a trouvé dix. Or une douzaine de coquilles (qui devront être corrigées au prochain tirage) dans un livre de 200 pages, voilà qui est bien peu et illustre au contraire la qualité matérielle de ce livre.

Ensuite, Réal Ouellet reproche à Ghislaine Legendre de ne pas avoir respecté l'usage que Louis Hémon faisait de la majuscule qu'il plaçait systématiquement aux mots désignant les mois et les points cardinaux. Cette décision est indiquée clairement dans l'avant-propos (p. IX) et elle me paraît tout à fait justifiée: les structures spatiales et temporelles du roman, comme aussi les thèmes du temps et de l'espace, sont assez fortement soulignées pour qu'il ne soit pas nécessaire de les mettre encore en évidence par un artifice graphique contraire à la norme typographique actuelle. Il s'agit d'un écart artificiel trop important pour être maintenu dans un roman qui se caractérise par sa très grande simplicité. Par ailleurs, l'édition en feuilleton dans Le Temps ne l'avait pas non plus maintenu, ce qui constitue une des normalisations tout à fait justifiables de cette édition journalistique.

Enfin, Réal Ouellet reproche à Ghislaine Legendre d'avoir régularisé la ponctuation erratique du manuscrit. Cette normalisation est pourtant réalisée dans la plupart des éditions critiques. L'important est qu'elle ne soit pas «refaite" et qu'elle soit conforme à l'esprit et à la lettre du texte de base. Or ce problème ne se pose que pour l'édition des textes antérieurs au XVIIle siècle, alors que les règles de ponctuation étaient différentes des règles que nous connaissons aujourd'hui et qui doivent être strictement respectées pour l'édition des textes en français moderne. Bien entendu, la ponctuation d'un texte est extrêmement importante et significative, mais jusqu'à preuve du contraire, il y a plus de chance que Ghislaine Legendre ait mieux respecté la ponctuation du manuscrit que les éditeurs qui non seulement en avaient refait la ponctuation, mais en avaient refait les phrases. Si tel n'est pas le cas, un «textologue» comme Réal Ouellet peut en faire la preuve en un tournemain.

Conclusion: deux coquilles et la régularisation de l'usage des majuscules et de la ponctuation discréditent complètement le travail de Ghislaine Legendre aux yeux sévères de Réal Ouellet. Par contre, l'introduction massive et absolument désordonnée des guillemets et des italiques par tous les autres éditeurs, cela lui paraît de simples «retouches» qu'il se contente de «déplorer». $A$ son avis, «pareils marquages se retrouvent fréquemment chez les écrivains québécois, aussi bien J.-A. Loranger que Savard.» Ben justement, Louis Hémon n'est pas un écrivain québécois, il n'est surtout ni Loranger ni Savard; il n'a pas collectionné de "beaux mots" pour les mettre entre guillemets dans un texte écrit en «bon français». Il a écrit Maria Chapdelaine dans une autre actualisation de la langue française, comme le feront beaucoup plus tard 
en effet les écrivains "québécois». Imaginons un instant l'apparition des guillemets à chaque page des romans de $V .-L$. Beaulieu et on obtiendra très précisément la version de Maria Chapdelaine qu'on lisait jusqu'à maintenant. Réal Ouellet fait preuve sur ce point non seulement d'une méconnaissance de la nature des langages littéraires, mais d'un des mécanismes les plus fondamentaux du texte écrit, l'usage des guillemets et des italiques.

\section{Prétendus problèmes d'édition critique}

Après l'invention des défauts, on ne sera pas surpris que Réal Ouellet soulève de prétendus problèmes d'édition critique dont il a justement la solution élégante sous les yeux. II écrit par exemple que «Les variantes prenant une telle importance, pourquoi n'a-t-on retenu que les «principales"? au nom de quels critères? Aucun textologue ne peut juger, à la place d'autrui, l'intérêt d'une variance textuelle». En bon textologue, Réal Ouellet aurait pu prendre quelques heures, comme je l'ai fait, pour confronter l'édition de Ghislaine Legendre avec celle qu'il avait sous la main (j'ai choisi l'édition Grasset du Livre de poche). II aurait pu faire alors le «compte-rendu» que j'ai déjà fait de l'édition sur ce point en constatant lui-même que toutes les variantes significatives avaient été retenues. En bon «textologue», j’ai déjà expliqué ce qu'il fallait entendre par une variante "significative» (qui a du sens, une signification). Si tel n'est pas le cas, Réal Ouellet en bon «textologue» peut en faire la preuve en un tournemain.

Ensuite, toujours en bon "textologue" et spécialiste de l'édition critique, Réal Ouellet voudrait un "protocole d'édition» (c'est-à-dire un exposé en bonne et due forme des principes de transcription du manuscrit) et «une liste complète des éditions avec leur filiation». Réal Ouellet a parfaitement raison: on ne trouve dans le livre de Ghislaine Legendre aucun exposé assommant, du genre universitaire, illisible pour tout autre qu'un "textologue». Mais un spécialiste de l'édition trouvera tout cela dans son livre. Il déduira lui-même comme un grand garçon le protocole d'édition de l'édition elle-même (s'il n'a pas entendu comme Réal Ouellet les deux communications qu'en a faites Ghislaine Legendre à l'intention des spécialistes) et il trouvera dans le relevé des variantes et l'avant-propos de Nicole Deschamps non seulement la filiation des onze éditions littéraires de Marie Chapdelaine (à l'exclusion de leurs innombrables dérivés en feuilleton, éditions pour adolescents et éditions de luxe), mais également la description des quatre états du texte.

En bon «textologue» par contre, Réal Ouellet aurait pu se demander pourquoi les «éditions" Fides de 1953, 1959 et 1965 n'avaient pas été retenues avec les onze éditions dépouillées par Ghislaine Legendre. II en aurait probablement conclu comme moi qu'il ne s'agissait pas d' "édition", mais simplement de «tirage» (les tirages des éditions de 1946, 1957 et 1960). Mais Réal Ouellet ne s'est pas posé cette question parce qu'il ne s'est tout simplement pas intéressé à l'édition critique dont il n'a pas rendu compte. II en aura simplement brandi le mythe. Voilà ce qu'on peut illustrer à l'aide du «problème» qu'il soulève à propos d'un des états du texte, la manipulation de l'édition J.-A. Lefebvre en 1916. 


\section{La manipulation de J.-A. Lefebvre}

Je cite d'abord Réal Ouellet qui cite Nicole Deschamps:

«Et y a-t-il seulement rapport entre les variances textuelles et le "mythe"? Comment peut-on déplorer que Maria ait été déformé par tant d'interprétations abusives et se permettre des généralisations aussi grossièrement abusives que la suivante: les «écarts», «introduits par Louvigny de Montigny, sont d'un grand intérêt sociologique parce qu'ils reflètent d'une façon simpliste l'idéal culturel de la société québécoise au début du 20 e siècle »? Au nom de quelle inspiration, bon sens ou dogmatisme, peut-on soutenir que Suzor-Côté a folklorisé, «mis entre guillemets la réalité québécoise décrite par Louis Hémon"? On tremble à la pensée de ce qu'aurait donné une lecture systématique de Maria par N. Deschamps.»

Même si la dernière phrase n'est pas très gentille, en bon professeur, je vais lui numéroter les faits sur lesquels. Nicole Deschamps a appuyé son affirmation, peut-être qu'il comprendra mieux. (1) Premièrement parce que les corrections de Louvigny de Montigny sont plus nombreuses que celles du journal Le Temps et qu'il corrige les propos de Monsieur le curé qui ne peut pas dire "Viens un peu par icitte, toué ». Un curé au Québec doit bien parler, ce que Louis Hémon ne savait pas. (2) Deuxièmement, parce que Louvigny de Montigny savait qu'on ne sacre pas au Québec et qu'on ne dit pas ciboire, mais vingt-gueux, ce que notre malheureux Louis Hémon, lui, ignorait. (3) Troisièmement parce qu'au Québec, non seulement les curés parlent bien et qu'on ne sacre pas, mais parce que tout le monde parle français international par cœur; par conséquent, les éditions de Montréal vont plus se conformer au français de Paris que les éditions parisiennes elles-mêmes. (4) Quatrièmement, parce que l'édition de J.-A. Lefebvre introduit au roman par deux préfaces de deux académiciens, un académicien de France (Émile Boutroux) et un académicien du Canada (Louvigny de Montigny lui-même). En plus, l'édition est accompagnée des illustrations académiques de Suzor-Côté. Bref, tout le monde allait savoir qu'il s'agissait d'un chef-d'cuvre et d'un modèle à imiter reconnu par toutes les académies. Voilà pourquoi la première édition montréalaise présente un intérêt sociologique évident (il n'y a aucun «bon sens ou dogmatisme» là-dedans): parce qu'il s'agit d'une «mise entre guillemets" de la société québécoise tout à fait contraire à l'esprit et à la lettre du roman de Louis Hémon.

Si Réal Ouellet demande encore "au nom de quoi» on peut se permettre des «généralisations aussi grossièrement abusives", ben coudon, je lui expliquerai encore avec des petits numéros les neuf autres raisons qu'on trouve en tête de l'exposé de Normand Villeneuve, pages 147 à 152 du Mythe de "Maria Chapdelaine». Mais je ferai cela après la classe pour ne pas retarder tout le monde.

\section{Virulence des propos et malveillance}

Après tout cela, c'est encore la virulence des propos qui étonne le plus. Car si Réal Ouellet n'a absolument aucune amélioration pratique à proposer 
pour aider Ghislaine Legendre à préparer une seconde édition, il ne manque pas de vocabulaire pour stigmatiser la "témérité" de la présentatrice qui aurait laissé entendre que "l'honnêteté et la science se trouvent dans sa seule édition" (c'est-à-dire celle de Ghislaine Legendre) réalisée "en dépit du sens commun" et dont l'avant-propos est plein de "généralisations grossièrement abusives». A quoi cela peut-il bien rimer? Et que penser de la note suivante à propos du manuscrit de Louis Hémon: "Ce tapuscrit, maintenant à l'Université de Montréal, a été confié à N. Deschamps en 1966 par Madame Lydia Louis-Hémon, qui n'est ni nommée ni remerciée. Pourtant la simple courtoisie...» D'abord, dans quel corridor de quelle université Réal Ouellet a-t-il appris que le manuscrit de Louis Hémon avait été «confié» et non «vendu» à I'Université de Montréal? Ensuite: mais de quoi se mêle-t-il? A quoi rime ce potinage sous la plume d'un universitaire?

Avec un tout petit peu de bonne volonté, je peux imaginer une explication très simple. Nicole Deschamps a remercié deux fois déjà Mme Hémon: la première fois dans la revue Études françaises en 1967 (volume III, $n^{\circ} .1, p .53$ ) et la seconde fois à la fin de son introduction à son édition de la Correspondance de Louis Hémon avec sa famille. Je suppose donc que par la suite le travail de Nicole Deschamps sur le mythe de Maria Chapdelaine risquait de ne pas plaire à Mme Hémon (comme il a déplu à Aurélien Boivin qui n'est pourtant pas le fils de Louis Hémon), de sorte que, par discrétion, Nicole Deschamps ne la nomme pas pour ne l'impliquer d'aucune manière dans un travail qu'elle n'approuve pas tout à fait ou qui risque seulement de lui déplaire. Il est bien possible que cette explication relève de mon imagination ou du roman, mais il est absolument certain que cela ne me concerne d'aucune manière et n'a aucun rapport avec la nature d'une édition critique. Personnellement, je suis peiné et choqué de cette malveillance et de ce manque de courtoisie que Nicole Deschamps ne méritait certainement pas.

Je dois dire en toute honnêteté que c'est d'abord à cause de cette malveillance que j'ai écrit la longue mise au point qu'on achève de lire. Par amitié et par admiration pour mes collègues et mes amies Ghislaine Legendre et Nicole Deschamps. Mais j'aurais dû être motivé seulement par l'honnêteté intellectuelle: parmi les dizaines de spécialistes de l'édition critique du Québec, il ne sera pas dit qu'il n'y en avait pas au moins un pour relever l'injure et ce mépris du travail d'autrui. Il est seulement dommage que ce ne soit pas un de ceux qui ont le plus de compétence et d'expérience.

\section{Le mythe de l'édition critique}

Comme Réal Ouellet n'est pas un incompétent (ce dont je ne doute pas), il faut qu'il se soit laissé emporter et qu'il ait forcé sa pensée pour en arriver à des conclusions absolument hors de proportion avec les «problèmes» qu'il soulève et qui sont sans rapport ni avec le travail d'édition critique, ni avec celle qu'a réalisée de manière pour le moins très correcte Ghislaine Legendre. Cette motivation, ce pourrait être de se porter à la rescousse de son collègue Aurélien Boivin (comme je le fais moi-même ici). Ce pourrait être aussi une 
manière de réagir à la publication du Mythe de "Maria Chapdelaine». Car quel professeur de littérature au Québec ne s'est pas enfoncé dans «cet imaginaire québécois", comme le fait Réal Ouellet dans les deux premiers tiers de son compte rendu, sans prendre en considération un seul instant, bien entendu, que son exercice de haute voltige plane au-dessus d'un roman pour adolescents. Mais il y a, me semble-t-il, une raison beaucoup plus importante, plus profonde et probablement inconsciente qui motive des propos si virulents: le mythe de l'édition critique.

Disons en quelques mots seulement qu'il existe plusieurs catégories d'éditions critiques. Celles-ci varient avec les textes édités (le genre, l'époque, la nature et l'état du texte), la perspective d'édition adoptée (linguistique, littéraire, philosophique ou historique, par exemple) et le public auquell'édition est destinée (ce qui est en général déterminé par la nature du texte: I'édition documentaire qui rassemble en transcription diplomatique de grandes collections de documents est radicalement différente de l'édition d'un texte littéraire que son auteur avait destiné à toute personne sachant lire le français). Et dans tous les cas, quels que soient le texte édité, la perspective adoptée et le public visé, l'édition critique peut comprendre deux travaux distincts: l'établissement du texte d'une part et l'annotation d'autre part. L'édition critique de Maria Chapdelaine par Ghislaine Legendre est l'établissement scientifique d'un texte littéraire destiné au grand public. Or cela est absolument contradictoire avec ce que beaucoup de spécialistes québécois de l'édition critique croient ou voudraient faire croire. II faut relire le texte de .Réal Ouellet que j'ai déjà cité, ses «Réflexions préliminaires sur l'édition d'un corpus québécois", pour comprendre qu'il n'y a qu'une seule et unique catégorie d'édition critique pour lui. II l'appelle l'«édition scientifique». Celle-ci doit comprendre dix rubriques (chronologie, introduction, histoire du texte, etc.). des piles de documents et je ne sais combien d'index. Cette édition doit bien entendu être réalisée par toute une équipe, durant plusieurs années et avec beaucoupbeaucoup d'argent. Au bout du compte, l'édition de Maria Chapdelaine fait environ 500 pages et coûte dans les quatre-vingt dollars. Et attention: il doit s'agir d'une édition scientifique et savante pour les spécialistes qui la «consulteront" (qui va lire un «instrument de bibliothèque»?) en bibliothèque ou la feront payer par... l'équipe en train de réaliser l'édition scientifique de Menaud Maître-Draveur. J'exagère peut-être un petit peu, mais il s'agit très exactement du mythe de l'édition critique que l'on trouve au cœur des comptes rendus de nos trois spécialistes sur l'édition de Ghislaine Legendre.

Je ne dis pas qu'il n'y a pas place pour l'édition critique annotée en domaine littéraire québécois, puisque je travaille moi-même dans une équipe qui entreprend d'éditer l'œuvre complète d'Hubert Aquin. Mais, personnellement, je ne crois pas que ce type d'édition soit justifié pour n'importe quelle œuvre littéraire, ni qu'il s'agisse du travail d'édition le plus important, le plus urgent et le plus utile au Québec. Par ailleurs, je ne déprécierai même pas mon travail en disant que celui de Ghislaine Legendre est à la fois plus discret et plus utile que le mien, parce que je me suis formidablement amusé à faire l'édition "scientifique» du Ragueneau du père Jacques Bigot (VLB Éditeur, 1979: je le propose aux bourses moyennement garnies!). 
Par contre, Ghislaine Legendre fait la preuve qu'une édition critique n'est pas un monstre sacré et qu'on peut produire rapidement et économiquement des éditions de la qualité de son Maria Chapdelaine. Un de nos plus beaux romans, Trente arpents de Ringuet, pourrait probablement être édité de cette manière avant six mois si la maison Fides (contre laquelle j'ai bien de la hargne, comme on le voit), avec ses conseillers universitaires, avait un minimum de compétence, de bonne volonté et, pour tout dire, un peu de respect pour ses lecteurs, puisque le manuscrit, si mes renseignements sont bons, est entre les mains des héritiers qui ne l'ont pas "confié" à l'Université de Montréal... Pour être utile aux lecteurs du Québec, il faudrait que l'édition critique soit autre chose qu'un mythe qui sert... à déprécier les éditions critiques qu'on réalise chez nous avec autant de compétence qu'ailleurs. L'édition critique de Maria Chapdelaine par Ghislaine Legendre n'est pas un beau rêve, comme l'édition critique de Trente arpents, mais un travail dont nous pouvons tous être fiers.

1. L'étude littéraire de Maria Chapdelaine:

Nicole Deschamps, "Lecture de Maria Chapdelaine" dans Études françaises, IV, 2 (mai 1968)2-167.

Pierre Pagé, «Maria Chapdelaine: un problème franco-québécois d'histoire littéraire" dans la Revue d'Histoire Littéraire de France, LXIX, 5 (octobre 1969), pp. 746-762.

2. Sociologie de la littérature: l'étude du mythe de Maria Chapdelaine:

Gabriel Boillat, «Comment on fabrique un succès: Maria Chapdelaine" dans la Revue d'Histoire Littéraire de France, LXXIV. 2 (mars 1974), pp. 223-225.

François Paré, "Maria Chapdelaine au Canada anglais: réflexions sur notre extravagance" dans Voix et Images, II, 2 (décembre 1976), pp. 265-278.

Nicole Deschamps, Raymonde Héroux et Normand Villeneuve, le Mythe de "Maria Chapdelaine", Les Presses de l'Université de Montréal, 1980.

3. L'érudition autour de Maria Chapdelaine: la constitution d'un savoir aussi inutile que tordant lorsqu'il est allégué à titre de «recherche universitaire»:

Aurélien Boivin, liste de toutes les traductions possibles et imaginables de Maria Chapdelaine dans le Saguenay-Lac-Saint-Jean célèbre Louis Hémon. Alma, les Éditions du Royaume, 1980 , pp. 43-52, repris dans l'édition destinée comme on sait aux «profanes»: Maria Chapdelaine, Montréal, Fides, collection «Bibliothèque québécoise», 1980, pp. 203-210. 